\title{
Editorial
}

\section{Decision-Making Modeling in Service Systems}

\author{
Zhimin Huang, ${ }^{1}$ Feng Yang, ${ }^{2}$ Desheng D. Wu, ${ }^{3}$ Victor Shi, ${ }^{4}$ and Alireza Amirteimoori ${ }^{5}$ \\ ${ }^{1}$ Robert B. Willumstad School of Business, Adelphi University, Garden City, Long Island, NY 11530, USA \\ ${ }^{2}$ School of Management, University of Science and Technology of China, No. 96 Jinzhai Road, Hefei 230026, China \\ ${ }^{3}$ School of Economics and Management, University of Chinese Academy of Science, No. 19 Yuquan Road, Beijing 100049, China \\ ${ }^{4}$ Lazaridis School of Business and Economics, Wilfrid Laurier University, Waterloo, ON, Canada N2L 6C1 \\ ${ }^{5}$ Department of Applied Mathematics, Islamic Azad University, Rasht, Iran
}

Correspondence should be addressed to Zhimin Huang; huang@adelphi.edu

Received 17 October 2016; Accepted 17 October 2016; Published 29 January 2017

Copyright (C) 2017 Zhimin Huang et al. This is an open access article distributed under the Creative Commons Attribution License, which permits unrestricted use, distribution, and reproduction in any medium, provided the original work is properly cited.

Global economic development has been exhibiting new characteristics. First, rapid developments in productivity make it possible for individuals to pursue more, as well as higher levels of, desires for novel services, diverse products, convenient communication, and innovative forms of entertainment. New service industries have emerged to fulfill such desires. Second, the innovation and development of new technologies, especially the maturity of the Internet and the appearance of IOT (Internet of Things), accelerate the growth of new service industries. Third, the pressure from environmental deterioration and depletion of resources force many countries to adjust industrial structures from manufacturing to service industries. As a result, service is playing a more significant role in modern society.

Nowadays, with the increasingly fierce market competition, compared with the same type of enterprises, it is difficult for an enterprise to get cheaper raw materials and labors, and it is also difficult to sell products at higher prices. In addition, the increasing scarcity of resource and environmental pressures force enterprises to lower resource consumption and environmental costs to provide products or services, which force enterprises to enhance the level of performance management. However, studies on operations, strategies, marketing, and financing of service systems remain scarce in the literature. Such a reality pushes us to organize a special issue aiming at discussing the decision-making methods in service systems which should be appropriate and applicable to improve the performance of service industries. It should be noticed that the performance indications of service systems are various in different industries, for example, friendliness of user interface in Internet service, profitability in banking service, timeliness in express logistics, and quality in health care.

The special issue has received 42 high-quality submissions. After a rigorous peer-review process, with an acceptance rate of about $30 \%$, thirteen papers have been accepted for publication. These accepted papers provide a variety of applications in service systems, and the authors have contributed towards combining the innovative methodology and mathematical analysis in solving real-world problems. The authors of these papers are from China mainland, Iran, Korea, Slovakia, and Taiwan. The service systems considered in these papers include health care, information technique and Internet, transportation, supply chain, retailing, power, and banking. This selection of papers reflects the diversity of research studies applying different decision-making models to improve the operations of service systems for different areas.

In this special issue, lots of decision-making methods are developed to improve the performance of different service systems. We classify these methods into four groups, including optimization, multiattribute or multiobjective decisionmaking, stochastic or fuzzy decision-making, and game theory based decision-making.

Optimization is one of the most important decisionmaking problems. It discusses the characteristics of the best choice of the decision problem and constructs the calculation method of the optimal solution. With the rapid development of computer and the improvement of the optimization method, lots of optimization problems in real world are 
solved. The optimization problems are widely found in economic planning, engineering design, production management, transportation, national defense, and other important areas. A representative study on the system optimization is to use mathematical programming to seek the best solution for a given objective function.

On the system optimization, in this special issue, $\mathrm{H}$. Zhu et al. develop a method for discovering and selecting cloud infrastructure services. With the rapid development of cloud computing, more and more cloud infrastructure service providers are providing large numbers of service instances. For cloud infrastructure services, the lack of a description standard and selection methods has hindered the services' discovering and choosing for users. H. Zhu et al. use a feature model to describe the configurable properties of a cloud infrastructure service and present methods of creating the service and demand models. Based on the description of the cloud infrastructure service, a systematic discovery and selection method for cloud infrastructure services is proposed. V. Modrak et al. have contributed a very interesting problem on the decision-making approach to select optimal platform of service variants. With the trend of mass customization, services are more abstract than products, and concepts for mass customization in manufacturing cannot be used directly in service without a methodical change. V. Modrak et al. focus on the development of a methodological framework to support decisions in a selection of optimal platform of service variants when compatibility problems between service options occurred. The approach is based on mutual relations between waste and constrained design space entropy. In addition, $\mathrm{Z}$. Li et al. have studied the medicine distribution route optimization problem for community health service institutions. Such a study is very important and fundamental. The optimization objective is defined as the overall cost which includes the refrigeration storage cost, vehicle fixed cost, and transportation cost. Considering the characteristics of medicine distribution for community health service institutions, $\mathrm{Z}$. Li et al. set up a distribution route optimizing model to minimize the overall cost. Finally, $H$. Lee et al. examine the effects of the emission cost on route choices of international container ships. The paper formulates a freight network model that captures decisions and interactions of ocean carriers and port terminal operators in the maritime freight transport system. Then, the emission cost is calculated based on an activity-based approach as a component of the ocean transportation cost function.

Multiattribute or multiobjective decision-making is a very important type of decision method for service system. The essential characteristic of service operations makes the importance of multiattribute or multiobjective decisionmaking more prominent. Compared with manufacturing operations, service operation has more objectives. Except the operational goals in manufacturing, such as reducing costs, saving manpower, reducing the waste, controlling the pollution, increasing profits, and increasing the flexibility, the targets of service operations also need to meet other goals, like improving customer satisfaction (or utility), speeding up the service response time and the intelligent level of service, accelerating product innovation speed, and others. Different kinds of targets are difficult to achieve at the same time. Thus the service operations can be characterized as multiattribute or multiobjective decision-making problems.

On the multiattribute or multiobjective decision-making problems, M. Mirzaei et al. have contributed towards solving a key theoretic problem in the representative multiattribute decision-making technique, namely, Data Envelopment Analysis (DEA). In multidimensional input/output space, the behavior of the firms can be analyzed by using efficient frontier or supporting surfaces of production technology. The piecewise linear frontier of DEA technology is not differentiable at the extreme points. M. Mirzaei et al. show that binding efficient supporting surfaces of an efficient point may be used to define different marginal rates of substitutions, and they get different marginal rates to each frontier point. T. Ding et al. propose multiple attribute decision-making based on cross-evaluation with uncertain decision parameters. The proposed approach assumes that all attribute weights are uncertain. It can overcome the drawback in prior research that the alternatives' ranking may be determined by a single attribute with an overestimated weight. In addition, the proposed method can also balance the mean and deviation of each alternative's cross-evaluation score to guarantee the stability of evaluation.

Stochastic or fuzzy decision-making usually pays attention to the decision problems where the attribution variables are not deterministic. Stochastic variable and fuzzy variable are the main types of indeterministic variables. Service system is in the state of evolution, and the property and variables are often in an unstable situation. If the distribution of some factors has certain regularity, stochastic distribution can be used to describe the state of the factors; if the distribution of the factors has no rules, then we can only use fuzzy mathematics to describe the state of the factors.

On the stochastic or fuzzy decision-making, W.-T. Pan and Y. Leu propose an analysis of bank service satisfaction based on quantile regression and grey relational analysis. With the grey relational analysis, they compare the effects of different variables on service satisfaction. Zhao develops an optimal replenishment policy for Weibull-distributed deteriorating items with trapezoidal demand rate and partial backlogging. To minimize the total cost per unit time, L. Zhao proposes an efficient solution procedure to determine the optimal replenishment and the optimal order quantity. In addition, Y. Chen et al. propose a robust service selection method based on uncertain QoS. QoS has become an important factor used to select the most appropriate service for users. The most prominent QoS-based service selection models only take the certain attributes into account. However, in the real world, there are a large number of uncertain factors. The method of Y. Chen et al. can help obtain a robust selection under uncertain situations.

Game theory mainly studies the interaction between the incentive structures. It is a mathematical method to study the phenomenon of competition among multiple decision makers. Game theory considers the individual's forecast behavior and the actual behavior in the game and studies their optimization strategies. Game-based decision-making has been one of the most important areas in decision science. 
In the area of game-based decision-making, J. Chen considers the strategic interactions under two sourcing strategies (single and dual sourcing) to improve the sourcing quality by considering a manufacturer that wants to outsource the manufacturing of two substitute products to external suppliers, which is a very innovative study. The authors find out that an appropriate proportion of quality investment sharing by the manufacturer can enable a cooperating strategy with a single supplier to be the dominant strategy. X. Sun et al. focus on the optimal service and price decisions of a supply chain with optional after-sale service in the supply chain composed of a manufacturer and a retailer. They derive the equilibrium decisions between the manufacturer and the retailer under two different cases. Crowdsourcing community is a very novel topic, and the motivation system is of great importance to develop the crowdsourcing community. J. Pang and $\mathrm{Z}$. Liu have contributed a precursory study on such a topic by using the principal-agent theory. They prove that enterprises can generate innovation plans of high quality and expected utility using the crowdsourcing community. Outsourcers can encourage high-quality people to join. Similarly, B. Luo et al. also focus on the design of incentive mechanism, while they aim at deflated performance manipulation in retail firms considering the ratchet effect and the reputation effect. They model a dynamic agency setting in which both the ratchet effect and the reputation effect are related to the store manager's compensation scheme. The dynamic decision makes the study of B. Luo et al. feasible and valuable.

We hope these papers will be helpful for readers to understand the wide use of decision-making methods in service systems and inspire their interest to further the exploratory research in this area, as well as its related topics.

\section{Acknowledgments}

We sincerely express our gratitude to the authors for their contributions and the reviewers for their time and effort in assessing the manuscripts. The support from NSFC (71322101 and 71631006) is also acknowledged.

Zhimin Huang Feng Yang Desheng $D . W u$ Victor Shi Alireza Amirteimoori 


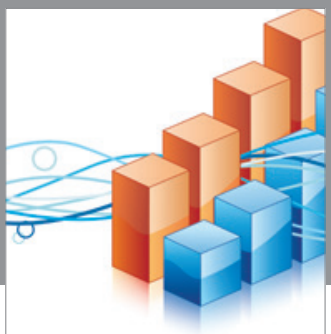

Advances in

Operations Research

vatem alat4

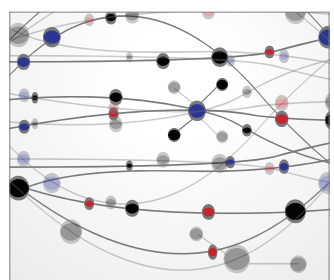

\section{The Scientific} World Journal
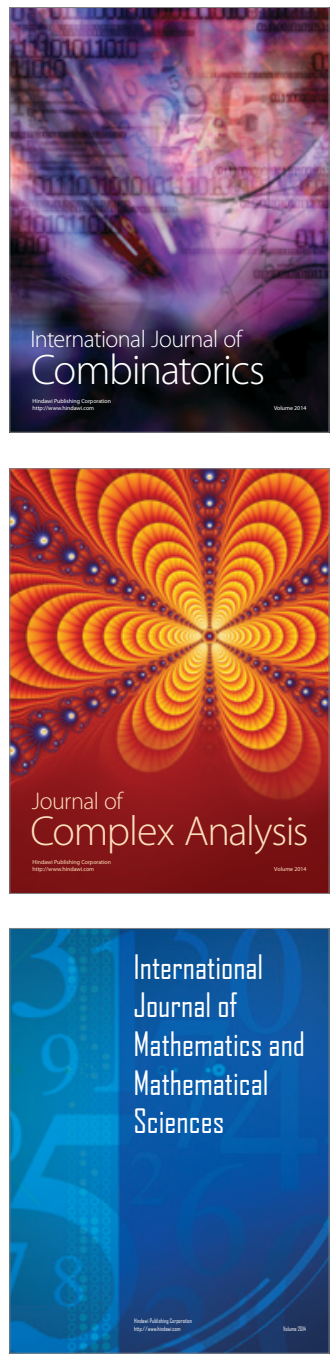
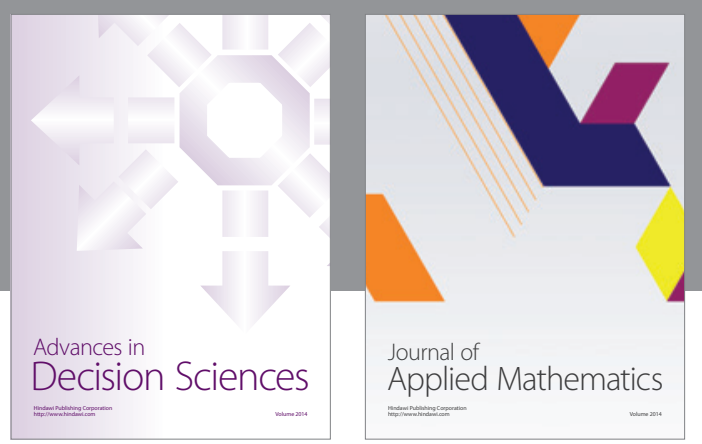

Algebra

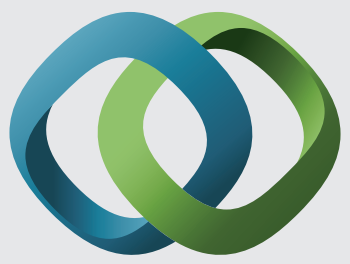

\section{Hindawi}

Submit your manuscripts at

https://www.hindawi.com
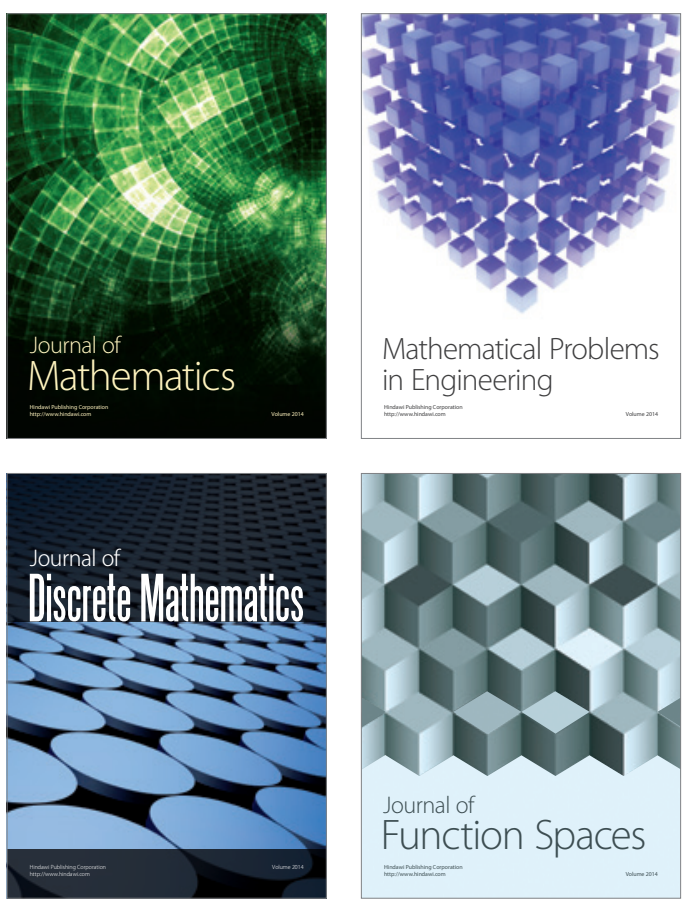

Mathematical Problems in Engineering
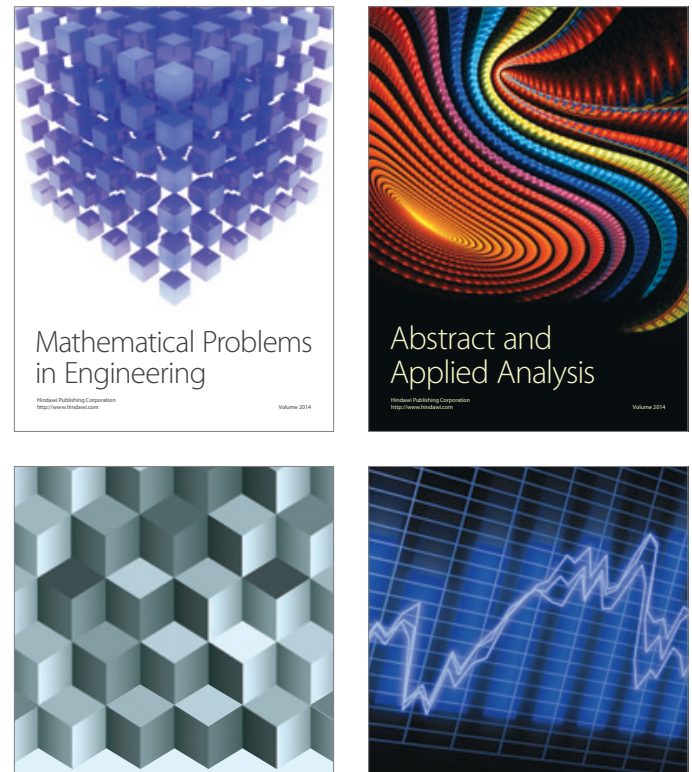

Journal of

Function Spaces

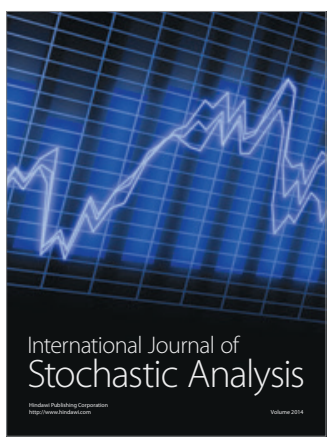

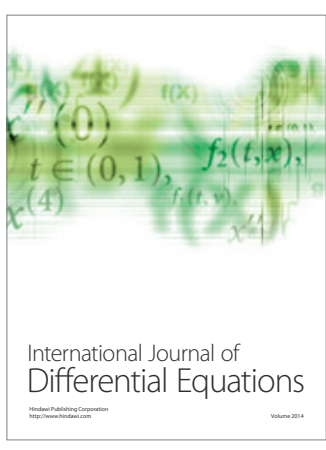
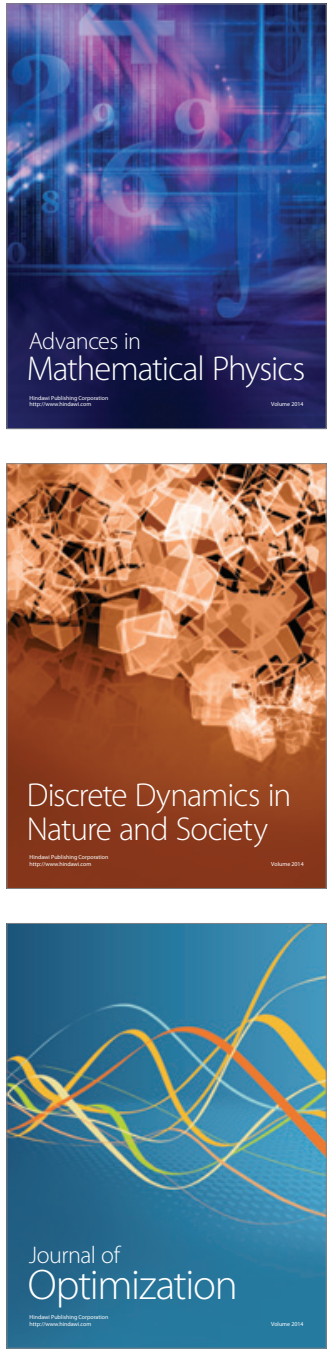